\title{
A Second Life for Electric Vehicle Batteries: Answering Questions on Battery Degradation and Value
}

\author{
Jeremy S. Neubauer, Eric Wood, and Ahmad Pesaran \\ National Renewable Energy Laboratory
}

\begin{abstract}
Battery second use-putting used plug-in electric vehicle (PEV) batteries into secondary service following their automotive tenure-has been proposed as a means to decrease the cost of PEVs while providing low cost energy storage to other fields (e.g., electric utility markets). To understand the value of used automotive batteries, however, we must first answer several key questions related to battery degradation, including: How long will PEV batteries last in automotive service? How healthy will PEV batteries be when they leave automotive service? How long will retired PEV batteries last in second-use service? How well can we best predict the second-use lifetime of a used automotive battery? Under the support of the U.S. Department of Energy's Vehicle Technologies Office, the National Renewable Energy Laboratory has developed a methodology and the requisite tools to answer these questions, including the Battery Lifetime Simulation Tool (BLAST). Herein we introduce these methods and tools and demonstrate their application. Under our assumed second use duty cycle of daily peak shaving, we have found that repurposed automotive batteries can last ten years or more in second use service when managed properly. In this role, capacity fade from automotive use has a much larger impact on second use value than resistance growth. Where capacity loss is driven by calendar effects more than cycling effects, average battery temperature during automotive service, which is often driven by climate, is found to be the single factor with the largest effect on remaining value. Installing hardware and software capabilities onboard the vehicle that can both estimate remaining battery capacity from in-situ measurements, as well as track average battery temperature over time, will thereby facilitate the second use of automotive batteries.
\end{abstract}

CITATION: Neubauer, J., Wood, E., and Pesaran, A., "A Second Life for Electric Vehicle Batteries: Answering Questions on Battery Degradation and Value," SAE Int. J. Mater. Manf. 8(2):2015, doi:10.4271/2015-01-1306.

\section{INTRODUCTION}

Increased market share of plug-in electric vehicles (PEVs) is one major strategy to reduce the nation's dependence on foreign oil and emissions of greenhouse gases by improving the overall fuel efficiency and cleanliness of vehicles in the United States. Accelerated market penetration of PEVs is presently restricted by the high cost of batteries, however. It has been estimated that an approximate $50 \%$ reduction in 2010 battery costs is necessary to equalize the economics of owning PEVs and conventionally fueled vehicles $[\underline{1}, \underline{2}]$.

Deployment of grid-connected energy storage systems, which could increase the reliability, efficiency, and cleanliness of the grid, is similarly inhibited by the cost of batteries. Over the past few years, mandates and incentives for energy storage have increased dramatically to overcome this barrier. For example, in 2010 the California legislature passed Assembly Bill 2514, which resulted in the California Public Utilities Commission releasing a procurement target for 1.3 gigawatts (GW) of energy storage in the state by 2020 [3] . Approximately $15 \%$ of this allotment has been planned for customer-sited, behind-the-meter storage [4]], further encouraged by California's self-generation incentive program, which offers up to $\$ 1.62$ per watt installed []].
Research, development, and manufacturing ramp-up efforts are underway to reduce battery costs by lowering material costs, enhancing process efficiencies, and increasing production volumes. However, it is also advisable to pursue increasing the total value of services provided by a battery over its lifetime. As PEV batteries may have substantial performance capability left at the end of their automotive service life, additional value could be extracted by committing them to other energy storage applications post automotive use, such as grid-connected services, including area regulation, backup power, or demand charge management. By extracting additional services and revenue from the battery in this post-vehicle application, the total lifetime value of the battery is increased, and the cost of the battery can be shared between the primary and secondary users. We refer to such strategies herein as Battery Second Use (B2U).

Many factors will determine the viability and economics of B2U strategies, from the costs of repurposing processes to the evolution of competing battery technologies. One of the most important factors in this equation is battery degradation in both the first (automotive) and second service lives. Herein we present the methodologies and tools developed by the National Renewable Energy Laboratory (NREL) under funding from the U.S. Department of Energy's Vehicle Technologies Office to provide the necessary understanding of 
battery degradation. We also present their application to a specific lithium ion chemistry (consisting of a nickel-cobalt-aluminum cathode and graphite anode) and example PEVs to explore likely outcomes.

\begin{abstract}
ANALYSIS
First we introduce our method for calculating battery degradation as a function of operating conditions. Next we discuss the motivation for and timing of battery replacement when in automotive service, leveraging our past studies that combined the battery degradation model with an automotive techno-economic analysis tool. Subsequently, we couple the battery degradation tool with an advanced long-term vehicle simulator to calculate expected battery wear during automotive service for different drive patterns, vehicle platforms, and climates. We then calculate the remaining life of these batteries when put to use under an anticipated second use duty cycle and discuss how this translates to remaining battery value. Finally, we explore practical methods for identifying second use value at the point of automotive retirement and its implications on the design of PEVs today.
\end{abstract}

\section{Modeling Battery Degradation}

Quantification of the relative amount of remaining performance of a battery at any point in time requires an understanding of battery degradation. Prediction of battery degradation to arbitrary operating conditions is currently a working area of research in the battery community. Many models that do so are empirically based, interpolating between or extrapolating from different sets of measured battery capacity and resistance data sets $[\underline{6}, \underline{7}, \underline{8}]$. These suffer from extensively large data needs and are often inadequate for extrapolating beyond the duration of the life test data upon which they are based. Alternatively, physics-based, first-principles models have been proposed, but can be exceedingly complex and limited in scope to a narrow range of operating conditions $[\underline{9}, \underline{10}, \underline{11}]$.

NREL has developed a semi-empirical life model that attempts to bridge this gap, offering a combination of increased confidence in interpolations and projections while maintaining simplicity of implementation and a basis in actual laboratory data [12]. It separately accounts for both capacity and resistance effects induced by cycling-based and calendar-based mechanisms. Cycling-based mechanisms specifically address the effect of charging and discharging the battery, while calendar-based mechanism address degradation that occurs even in the absence of current flow. Nonlinear effects of time, temperature, depth of discharge (DOD), and state of charge (SOC) are included. The specific model employed herein is fit to an extensive set of degradation data for a lithium-ion cell with a nickel-cobalt-aluminum cathode and graphite anode. We apply this model within an assumed 15-year vehicle life, at which point approximately $67 \%$ of all vehicles have been removed from service [13].

\section{Battery Replacement in Automotive Service}

An understanding of the time and state of health ( $\mathrm{SOH})$ of a PEV battery when it is removed from automotive service is also critical to calculating remaining battery performance. This requires knowledge of PEV battery replacement decisions, which can be made on multiple bases. In cases where the battery warranty ensures some minimum available performance criteria, the replacement decision may be straightforward. Vehicle leasing that entails similar performance guarantees would also be straightforward. At the time of writing, the 2014 Chevrolet Volt propulsion battery is warrantied to stay above $70 \%$ capacity during its 8-year/100,000-mile term [14]. The 2014 Nissan LEAF battery is similarly warrantied over a 5 -year/60,000-mile term [15]. Nissan also offers a battery warranty extension for $\$ 100 /$ month that can be extended for the total lifetime of the vehicle [16].

Where this is not the case and the vehicle is owned by its user, the majority of battery replacement decisions will be made by that user. The user's motivation for battery replacement may be (1) improved acceleration, (2) increased interior volume within the vehicle, (3) increased electric range of the vehicle, (4) decreased fuel costs, or (5) increased resale value. Our analyses suggest that vehicle acceleration is most likely limited by inverter and motor selection throughout the life of the vehicle, not the battery. Recovering additional interior volume following installation of a smaller battery would require considerable modification to a vehicle beyond battery replacement. Thus, motivations 1 and 2 are ruled out on the basis that these scenarios are expected to occur infrequently. Motivations 3, 4, and 5, on the other hand, are all viable, and all have quantifiable economic implications to the user (increased range leads to more miles driven on electricity rather than more expensive fossil fuels).

Thus, it is reasonable to assume that batteries will be replaced when either (1) a warranty (or similar) performance level is breached, or (2) there is an economic motivation for the user-owner to do so. Previous studies on battery degradation with NREL's Battery Ownership Model and the Battery Lifetime Analysis and Simulation Tool for Vehicles (BLAST-V)-advanced techno-economic simulators for PEVs employing the aforementioned battery degradation model-have found it likely that (1) PEV batteries will retain more than $70 \%$ of their original performance over the first 8 years of operation, and (2) replacing batteries within the anticipated 15 -year lifetime of a vehicle will not be economically justified $[\underline{17}, \underline{18}, \underline{19}]$. While there will be exceptions for select high-wear cases (particularly for high-mileage drivers in hot climates), they are likely to be a small percentage of the total B2U supply stock.

As such, the majority of B2U batteries should be expected to become available only at the end of a complete 15-year automotive service life, and the subsequent analyses herein shall be restricted to these cases.

\section{Battery Degradation in Automotive Service}

NREL's BLAST-V was applied to compute the SOH of PEV batteries following 15 years of automotive service. This highly detailed PEV simulator includes consideration of driver patterns and aggression, 
climate, cabin thermal dynamics, infrastructure, and many other factors to compute the wear incurred by the battery and utility delivered to the driver. More detail on the functionality of this tool can be found in the BLAST-V documentation [20].

Recent BLAST-V studies have found that driver patterns and climate have the largest effect on battery degradation among many other factors, including driver aggression, vehicle and battery thermal management systems, available infrastructure, etc. [1ㅗ $\underline{21}, \underline{22}]$. Thus BLAST-V is employed herein to predict battery degradation metrics for cold (Minneapolis, Minnesota), moderate (Los Angeles, California), and hot (Phoenix, Arizona) climates across a set of 91 year-long PEV-friendly drive patterns. These drive patterns are a subset of data recorded from the Puget Sound Regional Council's Traffic Choices Study [23] , processed per Neubauer and Wood [19], that yield a year-one vehicle miles traveled $\geq 8,000$ miles and a utility factor $\geq 80 \%$ when driven with a battery electric vehicle (BEV) with a 75-mile-range (BEV75), as presented in Figure 1.

Note that range of a BEV can vary greatly with operating conditions. Our definitions of nameplate range for PEVs are calculated via simulation and weighting of drive cycles per [24], which approximates the U.S. Environmental Protection Agency-rated range of a given vehicle. It is, however, common that our simulated PEVs achieve a greater than nameplate range as operating conditions allow.

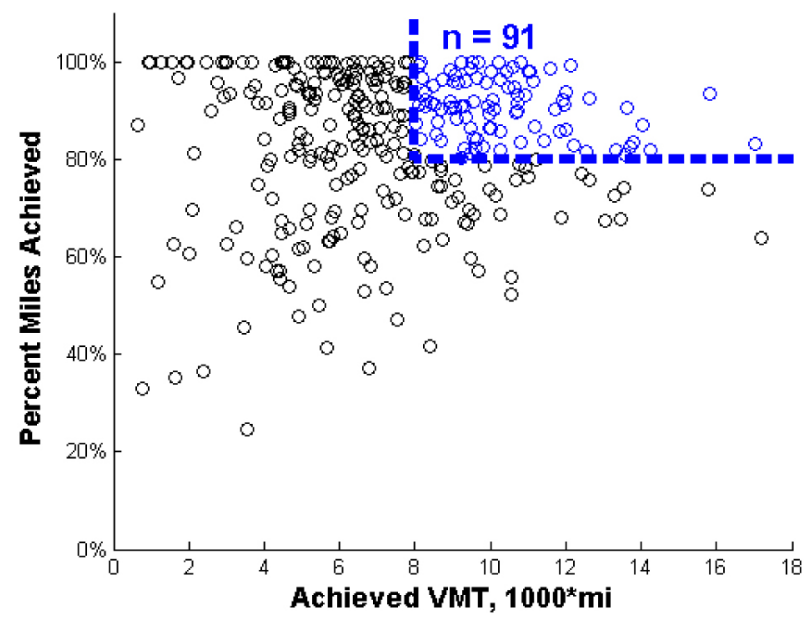

Figure 1. Utility factor vs. achieved vehicle miles traveled for selected drive patterns

For these drivers and climates, two different PEVs are simulated: (1) a midsize sedan BEV75, and (2) a midsize sedan plug-in hybrid electric vehicle (PHEV) with a 20-mile electric range (PHEV20). The BEV75 is equipped with a $22.1-\mathrm{kWh}$ battery that operates between $100 \%$ and $0 \%$ SOC. The PHEV20 is equipped with a $7.74-\mathrm{kWh}$ battery that operates in charge depleting mode between $100 \%$ and $20 \%$ SOC, then switches to charge sustaining mode. The PHEV battery is assumed to have $50 \%$ lower cell resistance than the BEV battery to reflect the higher relative power-to-energy ratio required of the PHEV20. Each vehicle employs a heat-pump cabin heater, a conventional cabin air conditioner, and an active battery cooling system. Further details of the battery and thermal systems models can be found in Neubauer and Wood [19].
Road load energy consumption is based upon simulation of NREL's Drive-Cycle Rapid Investigation, Visualization, and Evaluation (DRIVE) cycle, scaled for normal-aggression drivers and the average speed of the specific trip being simulated [19]. Auxiliary loadsbattery thermal management and cabin heating and cooling-are computed based on thermal simulations of the vehicle-battery system per Neubauer and Wood [19].

We assume that only at-home Level 2 charging $(6.6 \mathrm{~kW}$ alternating current) is available, and that the battery cooling system actively cools the battery when at the charger. All vehicles are simulated for 15 years, our assumed end of automotive service. The vehicle simulation specifications are given in Table 1.

Table 1. Vehicle Simulation Specifications

\begin{tabular}{|l|l|l|}
\hline Drivetrain & BEV75 & PHEV20 \\
\hline Vehicle type & Midsize sedan & Midsize sedan \\
\hline Battery size & 22.1 kwh & 7.74 kwh \\
\hline Typical electric range @ BOL & 75 miles & 20 miles \\
\hline Maximum SOC @ BOL & $100 \%$ & $100 \%$ \\
\hline Minimum SOC @ BOL & $0 \%$ & $20 \%$ \\
\hline Auxiliary equipment & Heat-pump cabin heater \\
\hline Charging infrastructure & Conventional cabin air conditioner \\
\hline BOL = beginning of life & $\begin{array}{l}\text { At-home Level 2 (6.6 alternating current, } \\
93 \% \text { efficiency) }\end{array}$ \\
\hline & Battery cooling active at charger \\
\hline
\end{tabular}

The resultant battery $\mathrm{SOH}$ data are presented in Figures $2, \underline{3}, \underline{4}, \underline{5}$ for all drive patterns. Table 2 presents the median $\mathrm{SOH}$ values taken from all of the drive patterns.

These data show that for both the BEV75 and PHEV20, capacity loss is dominated by calendar effects (Q1), resulting in losses of $25 \%$ to $35 \%$ after 15 years of automotive life, depending on climate and driving pattern. The effect of climatic differences between Minneapolis and Los Angeles is significant, resulting in 4\% more Q1 capacity loss in Los Angeles. The difference between Phoenix and Los Angeles is only 1\%, however, presumably due to the presence of the active cooling system above $20^{\circ} \mathrm{C}$. Median Q1 capacity losses are approximately $4 \%$ higher in the PHEV20 than the BEV75. While the cycling effect on capacity (Q2) is much greater (approximately $12 \%$ ) in PHEVs than in BEVs due to the increased frequency of high DOD cycling, this has no immediate impact on battery performance or value, as the total capacity loss is determined by the greater of Q1 and Q2, not the sum.

Calendar effects on resistance (R1) are nearly identical for the BEVs and PHEVs. In all cases, the 25th to 75th percentile spread is extremely small. For the BEV, it is likely that the calendar effects on resistance are significantly larger than the cycling effect (R2); however, for the PHEV this trend is reversed: cycling effects (R2) are much more likely to dominate total resistance growth. As such, total resistance growth (the sum of R1 and R2) is much larger in the 
PHEV20 than the BEV75. The observed effect of climate on resistance growth is similar to that of capacity fade: a significant difference exists between Minneapolis and Los Angeles, but a minimal difference exists between Los Angeles and Phoenix due to the set points of the active battery cooling system.
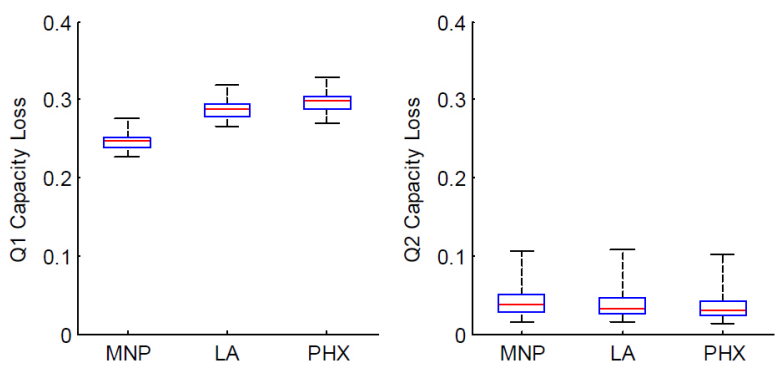

Figure 2. Box plot of BEV75 battery capacity loss due to calendar effects (Q1) and cycling effects (Q2); note that the total capacity fade is the greater of Q1 and Q2
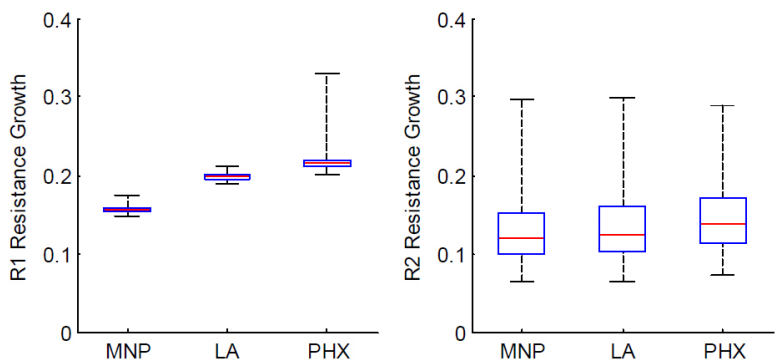

Figure 3. Box plot of BEV75 battery resistance growth due to calendar effects (R1) and cycling effects (R2); note that total resistance growth is the sum of $\mathrm{R} 1$ and $\mathrm{R} 2$
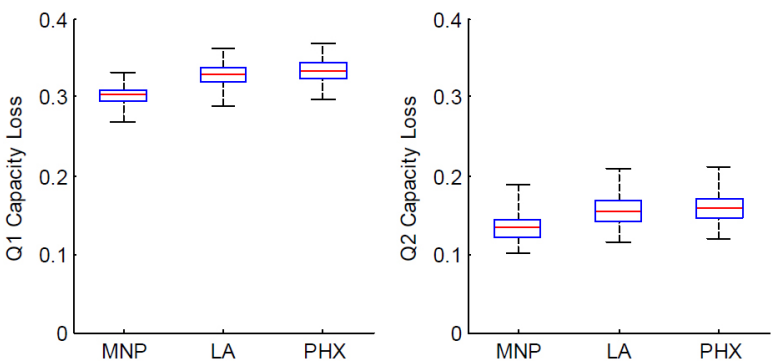

Figure 4. Box plot of PHEV20 battery capacity loss due to calendar effects (Q1) and cycling effects (Q2); note that the total capacity fade is the greater of Q1 and Q2
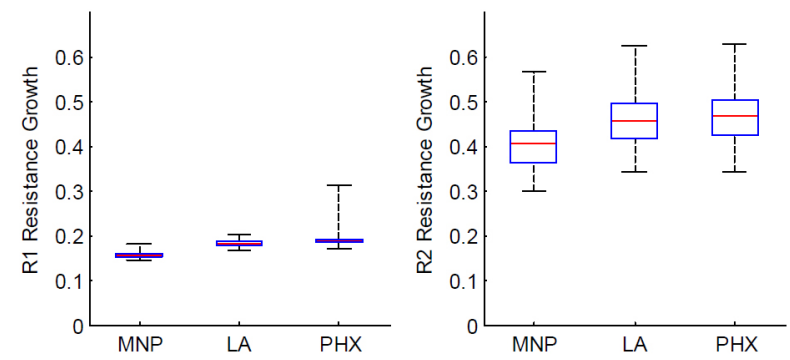

Figure 5. Box plot of PHEV20 battery resistance growth due to calendar effects (R1) and cycling effects (R2); note that total resistance growth is the sum of R1 and R2
Table 2. Median Capacity Loss and Resistance Growth Due To Across Driving Patterns

\begin{tabular}{|l|l|l|l|l|}
\hline \multirow{2}{*}{$\begin{array}{l}\text { VOH } \\
\text { Vehicle }\end{array}$} & Minneapolis, MN & $\begin{array}{l}\text { Los Angeles, } \\
\text { CA }\end{array}$ & Phoenix, AZ \\
\hline $\begin{array}{l}\text { Capacity, } \\
\text { Calendar (Q1) }\end{array}$ & BEV75 & $24.7 \%$ & $28.8 \%$ & $29.8 \%$ \\
\cline { 2 - 5 } & PHEV20 & $30.2 \%$ & $32.9 \%$ & $33.4 \%$ \\
\hline $\begin{array}{l}\text { Capacity, } \\
\text { Cycling (Q2) }\end{array}$ & BEV75 & $3.7 \%$ & $3.2 \%$ & $2.9 \%$ \\
\cline { 2 - 5 } & PHEV20 & $13.3 \%$ & $15.5 \%$ & $15.9 \%$ \\
\hline $\begin{array}{l}\text { Resistance, } \\
\text { Calendar (R1) }\end{array}$ & BEV75 & $15.5 \%$ & $19.9 \%$ & $21.4 \%$ \\
\cline { 2 - 5 } & PHEV20 & $15.5 \%$ & $18.2 \%$ & $18.7 \%$ \\
\hline $\begin{array}{l}\text { Resistance, } \\
\text { Cycling (R2) }\end{array}$ & BEV75 & $12.0 \%$ & $12.4 \%$ & $13.8 \%$ \\
\cline { 2 - 5 } & PHEV20 & $40.6 \%$ & $45.9 \%$ & $46.7 \%$ \\
\hline
\end{tabular}

\section{Predicting Remaining Battery Performance}

While BLAST-V provides predictions of battery capacity fade and resistance growth to the end of automotive service, these numbers alone are not wholly indicative of remaining battery performance. For example, the loss of $25 \%$ of initial capacity does not necessarily imply that $25 \%$ of the battery's value is lost: the battery could operate for only a few more cycles before becoming completely unusable, which would imply a much larger loss of value, or it may continue operate with minimal additional degradation for decades, which would imply a much smaller loss of value. As these examples illustrate, the remaining performance is also dependent on the number of remaining cycles and calendar time.

For the purposes of this study, where the interest in remaining value is primarily economic, an economic approach to the calculation of the remaining value is called for, such as Neubauer et al.'s Present Value of Throughput (PVT) method [25]. This method assumes that the battery's owner accumulates $D$ dollars for every kilowatt-hour of throughput processed by the battery today, that $D$ is expected to escalate at $2.5 \%$ per year, and that the owner's discount rate for future cash flows is $10 \%$ per year. Then, if the battery is operated with any arbitrary monthly throughput of $x_{i}$, for which it is known that the battery will last $m$ months, the present value of the battery's remaining service life is given by Equation 1 .

$$
P V T=\sum_{i=1}^{m} \frac{(1+0.025)^{(i-0.5) / 12}}{(1+0.10)^{(i-0.5) / 12}} D x_{i}
$$

Definition of the health factor, $k_{H}$, in Equation 2 as the ratio of remaining $P V T$ of a used automotive battery $\left(P V T_{U}\right)$ to that of a new battery providing identical service $\left(P V T_{N}\right)$ allows comparison of the value of a used battery relative to a new one. It is important to recognize that "identical service" means that the value of throughput $(D)$ is the same (thus the value for $D$ need not be specified). The number of months of service $(m)$ and the annual throughput from the battery $\left(x_{i}\right)$ are anticipated to be different for $P V T_{N}$ and $P V T_{U}$. For example, one may find that $P V T_{N}$ is optimized when a new battery is 
operated at a high annual throughput (implying a high DOD) over 10 years, while a used battery may be required to operate at a lower annual throughput (restricted by available energy) and may only be capable of sustaining such operation for five years.

$$
k_{H}=P V T_{U} / P V T_{N}
$$

A simplified peak-shaving duty cycle consisting of a constant-power, two-hour discharge in the afternoon and a six-hour, constant-power, constant-voltage charge overnight performed 252 days per year was assumed to compute $P V T_{N}$. This is a reasonable duty cycle for a battery performing in a generic peak-shaving role, which is a reasonable expectation for a second use battery. An average temperature of $10^{\circ} \mathrm{C}$ above the U.S. national average ambient temperature of $11.16^{\circ} \mathrm{C}$ was assumed [26] to conservatively represent the combined effects of the battery container, heat generation during discharge, and solar irradiation if the battery is located outside. This effective temperature may also be representative of locating the battery inside a climate-controlled facility with moderate cooling. We select a maximum SOC of $100 \%$.

These conditions were simulated for DODs of $40 \%, 50 \%, 60 \%$, and $70 \%$, and $P V T_{N}$ was computed for each based on the resultant service life $(m)$ to select a near optimal DOD. End of service life was defined as the point at which the battery could not sustain the defined duty cycle at or above the minimum allowed cell voltage and $0 \%$ SOC. Data for the BEV75 cells are shown in Figure 6, where $P V T_{N}$ has been normalized to the best performer in this set. The use of a $50 \%$ DOD, yielding a 16.9-year lifetime, maximizes PVTN. Simulation of the lower-resistance PHEV20 cell yielded similar results (not shown), but the battery lasts slightly longer (17.2 years at 50\% DOD). Thus, a $50 \%$ DOD for each cell is employed as the optimal condition for the purpose of $P V T_{N}$.

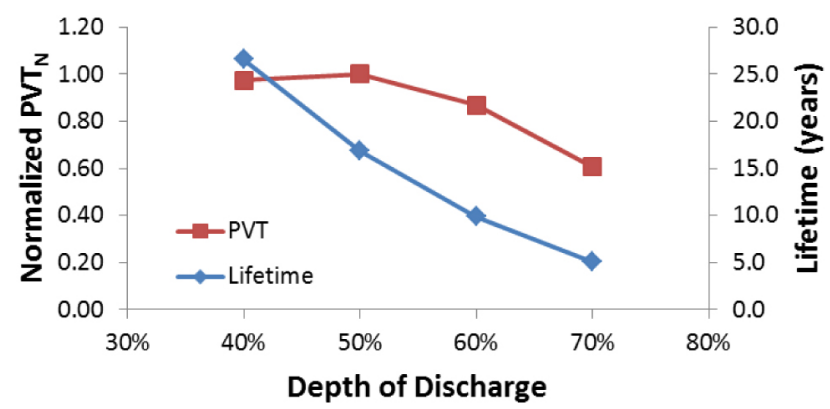

Figure 6. Lifetime and $P V T_{N}$ for a new BEV75 battery operated at various DOD for a simplified peak-shaving duty cycle

Using the resultant lifetimes for the BEV75 and PHEV20 cells at $50 \%$ DOD, we can derive an equation for $P V T_{N}$ as a function of $D$ and beginning of life (BOL) capacity, $Q_{B O L}$ in Equations 3 and $\underline{4}$, assuming 30.4 days per month:

$$
P V T_{N, B E V}=\sum_{i=1}^{202} \frac{(1+0.025)^{(i-0.5) / 12}}{(1+0.10)^{(i-0.5) / 12}} D x_{i}=1797 D Q_{B O L}
$$

$$
P V T_{N, \text { PHEV }}=\sum_{i=1}^{122} \frac{(1+0.025)^{(i-0.5) / 12}}{(1+0.10)^{(i-0.5) / 12}} D x_{i}=1815 D Q_{B O L}
$$

Next, batteries removed from automotive service were simulated to a similar peak-shaving cycle employed for $P V T_{N}$ to calculate the sustainable second use service life. DOD selection in second use was limited by two factors. First, large DODs were limited by capacity lost in automotive service. For example, a battery that has already lost $30 \%$ of its initial capacity cannot cycle at greater than a $70 \%$ DOD, as DOD is referenced to BOL battery capacity. Further, if this battery were cycled at $70 \% \mathrm{DOD}$, it would only be able to deliver this full cycle once as the battery continues to age. Second, it is unreasonable to employ excessively low DODs that result in extremely long battery lifetimes-these batteries will already have served 15 years in an automobile, and they are unlikely to have been designed to substantially exceed the vehicle's lifetime. Therefore, other mechanisms not accounted for in the battery degradation model (e.g., corrosion, failure of cell seals, fatigue of electrical connections, long-term electrochemical effects not yet witnessed in the underlying data) may become the primary pack failure mode if the second use lifetime becomes too large. For these reasons, investigations herein are limited to $50 \%$ and $60 \%$ DOD scenarios and a maximum 10 -year second-use battery life.

After simulating the second use lifetime data, $k_{H}$ was calculated. Because the duty cycle is the same as that employed for computing the reference $P V T_{N}$, Equations $1, \underline{2}, \underline{3}, \underline{4}$ can be combined and simplified to Equations $5, \underline{6}, \underline{7}, \underline{8}$.After simulating the second use lifetime data, we now turn to calculating $k_{H}$. Because our duty cycle is the same as that employed for computing our reference $P V T_{N}$, we can combine and simplify Equations $1, \underline{2}, \underline{3}, \underline{4}$ to yield Equations $5, \underline{6}$, $\underline{7}, \underline{8}$.

$$
\begin{aligned}
& k_{H, 60 \%, B E V 75}=\frac{0.6 * 30.4}{1797} \sum_{i=1}^{m} \frac{(1+0.025)^{(i-0.5) / 12}}{(1+0.10)^{(i-0.5) / 12}} \\
& k_{H, 60 \%, \text { PHEV } 20}=\frac{0.6 * 30.4}{1815} \sum_{i=1}^{m} \frac{(1+0.025)^{(i-0.5) / 12}}{(1+0.10)^{(i-0.5) / 12}}
\end{aligned}
$$

$$
k_{H, 50 \%, B E V 75}=\frac{0.5 * 30.4}{1797} \sum_{i=1}^{m} \frac{(1+0.025)^{(i-0.5) / 12}}{(1+0.10)^{(i-0.5) / 12}}
$$

$$
k_{H, 50 \%, \text { PHEV } 20}=\frac{0.5 * 30.4}{1815} \sum_{i=1}^{m} \frac{(1+0.025)^{(i-0.5) / 12}}{(1+0.10)^{(i-0.5) / 12}}
$$


The results of the second use lifetime simulations and health factor calculations for both vehicles and DODs are presented in Figures 7, $\underline{8}, \underline{9}, \underline{10}$. Median second use lifetimes and health factors across first use drive cycles are tabulated in Table 3 . As with the automotive simulation results, the differences in predicted second use lifetimes and health factors between the batteries removed from service in Los Angeles and Phoenix were much smaller than the differences between the Los Angeles and Minneapolis batteries. Batteries from the PHEV20s were found to have considerably worse second use performance than those removed from BEV75s due to a combination of higher resistance growth and cycling-based capacity fade from automotive service. However, where the 10-year maximum B2U lifetime restriction comes into play (which occurs quite frequently for the $50 \%$ DOD cases), the impact of first-use climate is greatly reduced as most batteries are predicted to exceed the 10-year limit. Finally, the seemingly small difference in second use DOD (50\% vs. $60 \%$ ) was observed to have a large effect on second use lifetime and health factor: where the 50\% DOD lifetime does not exceed our 10-year simulation limit, the lifetime and health factor of the $60 \%$ DOD case is approximately doubled.
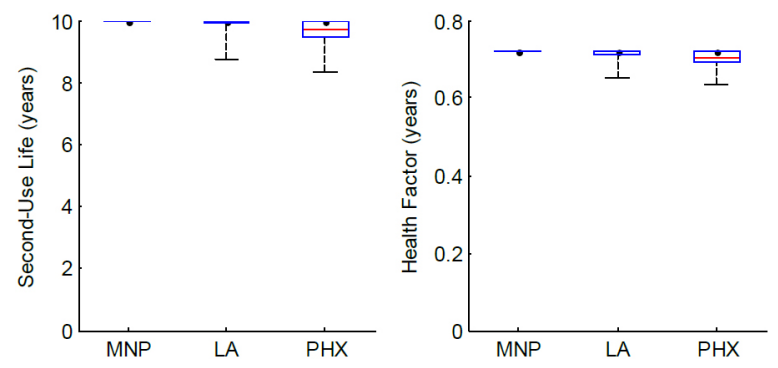

Figure 7. Predicted second use service life and health factor, 50\% DOD, BEV75; many cases reached the 10-year simulation limit
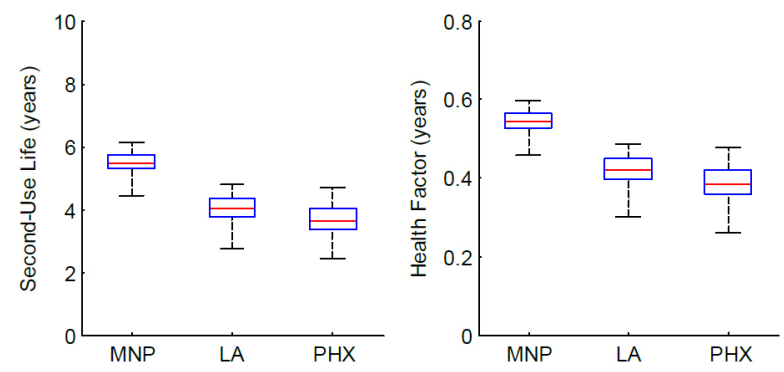

Figure 8. Predicted second use service life and health factor, 60\% DOD, BEV75; no cases reached the 10-year simulation limit
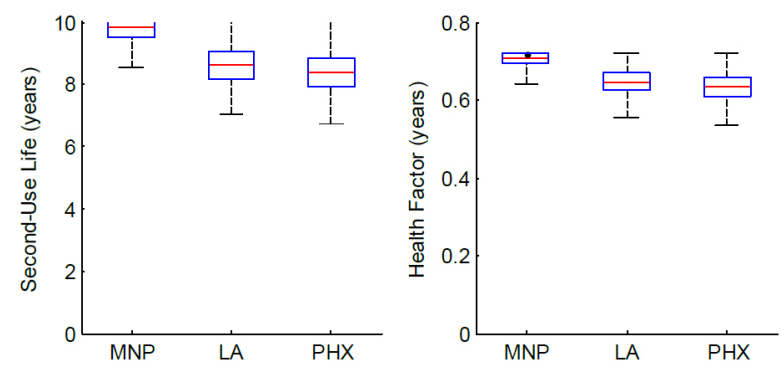

Figure 9. Predicted second use service life and health factor, 50\% DOD, PHEV20; many Minneapolis cases reached the 10-year simulation limit; few Los Angeles and Phoenix cases reached the 10-year simulation limit
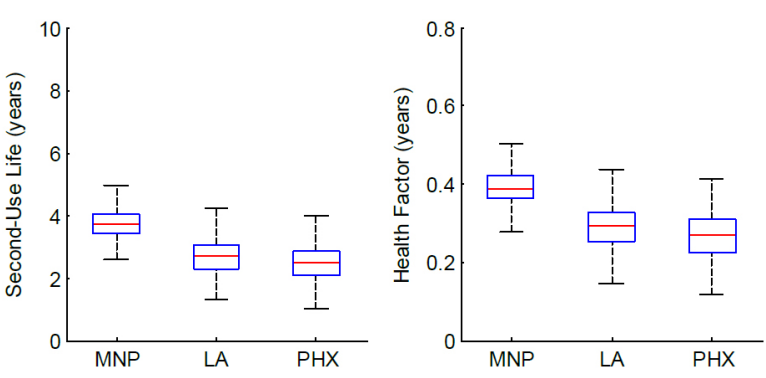

Figure 10. Predicted second use service life and health factor, 60\% DOD, PHEV20; no cases reached the 10-year simulation limit

Weighting the results from these vehicle platforms and climates to represent the second use performance of all PEV batteries from across the United States is challenging. Doing this properly entails not only forecasting the distribution for PEV deployments, but also forecasting the changing climate, which is beyond the scope of this study. Thus, on the simple basis that PEVs will be most successful in moderate climates, the predicted second use lifetimes and health factors from Los Angeles were employed for further evaluation. Both the $50 \%$ and $60 \%$ DOD cases will be retained for further study due to the significant differences in lifetime and value that result.

Table 3. Median Second Use Lifetimes and Health Factors for BEV75 and PHEV20

\begin{tabular}{|c|c|c|c|c|c|}
\hline DOD & Parameter & Vehicle & $\begin{array}{l}\text { Minneapolis, } \\
\text { MN }\end{array}$ & $\begin{array}{l}\text { Los } \\
\text { Angeles, } \\
\text { CA }\end{array}$ & $\begin{array}{l}\text { Phoenix, } \\
\text { AZ }\end{array}$ \\
\hline \multirow{4}{*}{$50 \%$} & \multirow{2}{*}{$\begin{array}{l}\text { Second Use } \\
\text { Lifetime } \\
\text { (years) }\end{array}$} & BEV75 & $10^{*}$ & $10^{*}$ & $9.7^{*}$ \\
\hline & & PHEV20 & $9.8^{*}$ & 8.6 & 8.4 \\
\hline & \multirow{2}{*}{$\begin{array}{l}\text { Health } \\
\text { Factor, } k_{H}\end{array}$} & BEV75 & $0.72^{*}$ & $0.72^{*}$ & $0.70^{*}$ \\
\hline & & PHEV20 & $0.71^{*}$ & 0.65 & 0.63 \\
\hline \multirow{4}{*}{$60 \%$} & \multirow{2}{*}{$\begin{array}{l}\text { Second Use } \\
\text { Lifetime } \\
\text { (years) }\end{array}$} & BEV75 & 5.5 & 4.0 & 3.6 \\
\hline & & PHEV20 & 3.7 & 2.7 & 2.5 \\
\hline & \multirow{2}{*}{$\begin{array}{l}\text { Health } \\
\text { Factor, } k_{H}\end{array}$} & BEV75 & 0.42 & 0.33 & 0.30 \\
\hline & & $\begin{array}{l}\text { PHEV20 } \\
\end{array}$ & 0.39 & 0.29 & 0.27 \\
\hline
\end{tabular}

\section{Identifying Remaining Battery Performance}

While we previously discussed the topic of predicting remaining battery performance for the purpose of our forward-looking analyses, the repurposer will need to identify the remaining battery performance for each battery they consider for purchase. The first question that arises is which $\mathrm{SOH}$ parameter is most important to identify. To answer this question, we present the predicted second use battery lifetimes for our previous simulations as a function of the battery SOH parameters at the end of automotive service in Figure 11. The lack of correlation between second use lifetime and R1, R2, and Q2 stands in stark contrast to the extremely consistent and linear correlation between second use lifetime and Q1-capacity fade due primarily to calendar effects. We see the same correlation between second use lifetime and total capacity fade, as all of our automotive simulations resulted in dominant Q1 fade. 

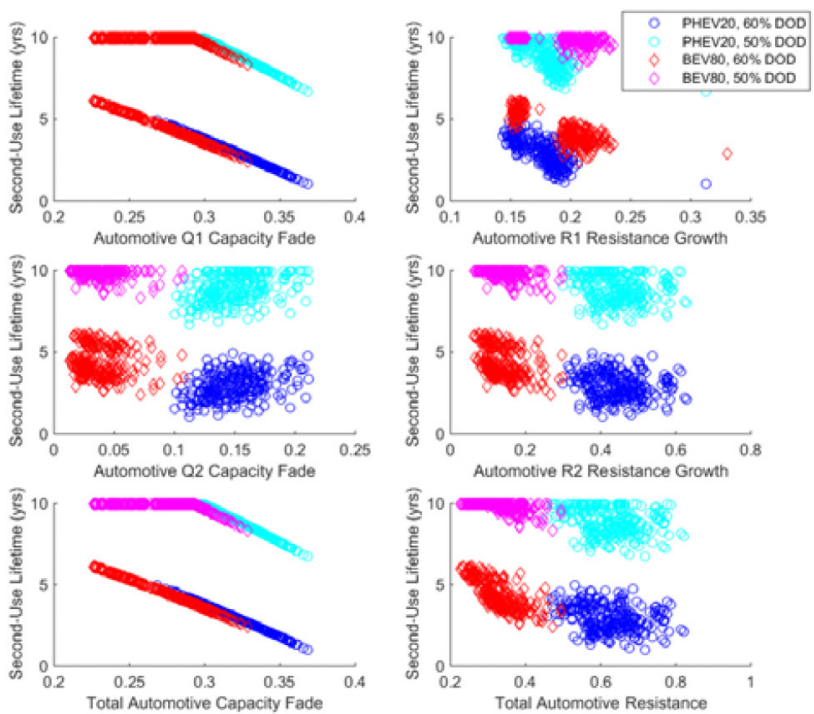

Figure 11. Comparison of second use lifetime to battery degradation at end of automotive service

Thus, identifying Q1 appears to be crucially important to diagnosing the second use value of a retired automotive battery. This could be accomplished by three different means: (1) directly measuring battery capacity at the point of repurposing, (2) applying computational methods to duty cycles recorded near the end of a battery's automotive service life, or (3) by recording and reporting relevant metrics from automotive service that correlate strongly to Q1 capacity fade. The first method is well known and requires no additional capabilities be added to the vehicle; however, it requires specialized testing equipment and a relatively long testing duration. Thus, it is viable for the repurposer to perform after a purchase has been made, but not before. The latter two are not well developed and do require some additional capabilities be added to the vehicle at BOL. These latter two methods are discussed briefly below.

\section{In-Situ Analysis of Drive Cycles and Charge Events to Identify Q1 Capacity}

As applied in this study, mathematical battery models can be used to predict the voltage, current, temperature, and SOC response of a battery to a specific duty cycle. However, this problem may also be inverted: knowledge of a battery's response to a duty cycle can be applied to create a model. While many of our battery models are created this way using purpose-designed duty cycles, it is also possible to build models using arbitrary duty cycles. Thus, if a sufficient set of battery response parameters were monitored and recorded on-board a vehicle, a model of that battery could be built from regular field use of the vehicle without the need for specialized equipment, visits to a service center, etc. Done properly, this model could extract the $\mathrm{SOH}$ parameters necessary to evaluate a battery for B2U. The data could be applied not only to second-use valuation, but also range estimation, vehicle-to-grid assessment, and other purposes throughout the battery's automotive life. It is likely that the benefits of these automotive life services-perhaps range estimation alone-are sufficient to motivate the inclusion of such technology by OEMs.
NREL has developed and applied such algorithms on a limited basis to BEVs operating in the field. Battery duty cycles harvested from large data sets of in-use operation provide time series histories of pack and cell-level current and voltage. These data are applied to a battery electrical model that considers zero-order equivalent circuit dynamics and a single-particle model of electrode concentration gradients (used to describe transient voltage relaxation). The modeled battery voltage is compared to the historical data, and a constrained non-linear optimization algorithm is used to minimize the root mean square of model error (usually achieving root mean square error values of tens of millivolts per cell). Error is minimized by updating model parameters such as pack capacity, bulk resistance, initial thermodynamic state of charge, and multiple diffusion coefficients. An example comparison of modeled and measured battery response is shown in Figure 12.

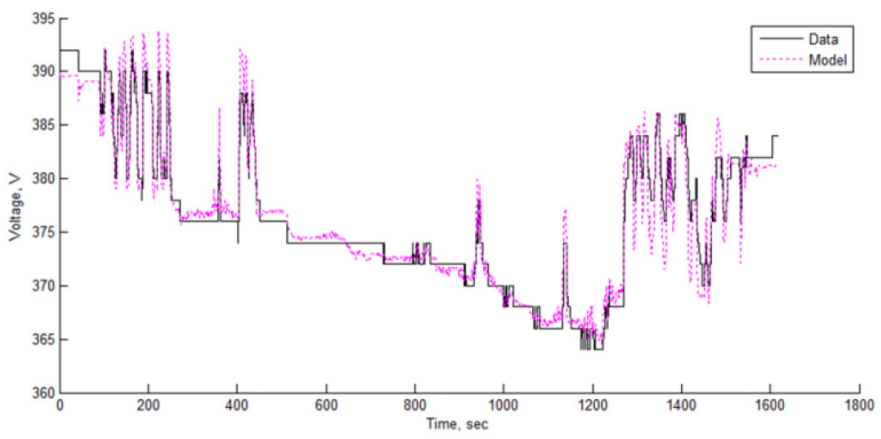

Figure 12. Comparison of second use lifetime to battery degradation at end of automotive service

Following optimization of the model over each individual drive cycle, estimated parameters used to describe pack available energy and power are reported through time and compared to controlled performance tests conducted by NREL engineers in the field as available.

Figure 13 shows an example of fitting battery capacity and resistance for individual cells in six different battery electric vehicles over an approximate 2.5-year period. Applying these methods at the cell level offers the ability to identify individual cells with anomalous capacity or resistance parameters in addition to quantifying total pack performance.
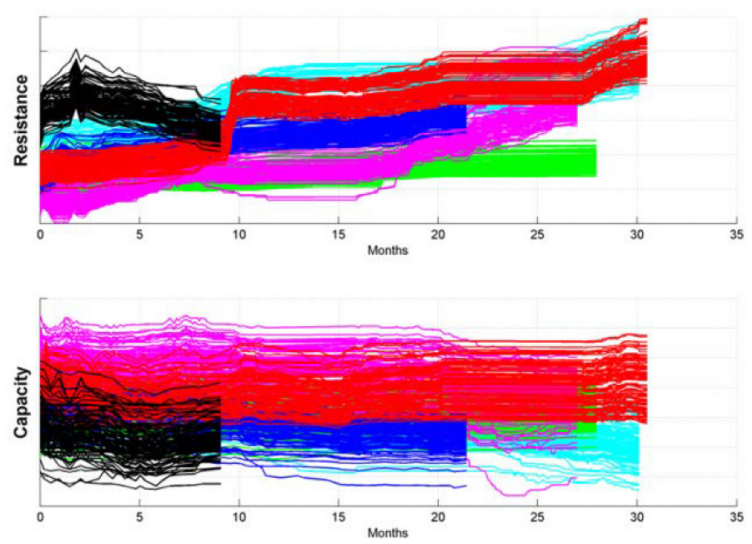

Figure 13. Example results of cell-level capacities and resistances calculated from field data from six BEVs 
To date, this procedure has been applied to a small number of vehicle histories to develop and validate the technique. While the results of these methods have been encouraging, there is considerable room for improvement. Beyond advancing model fidelity and fitting algorithms, inclusion of battery response to charging events is expected to be both the easiest and most valuable addition to these methods (due to onboard hardware and software limitations, charging events were not available to NREL researchers in the work noted in these examples). Such low-rate, constant-current duty cycles, often terminated at a relatively precise final SOC and with considerable resting periods thereafter, will greatly improve the accuracy of capacity identification in particular-the critical component for second use battery valuation. Modifications to the charge protocol such as occasional charge interruptions to assess resistance or open-circuit voltage could also be implemented by the battery management system to further increase accuracy of $\mathrm{SOH}$ assessments.

The simplicity and potential for high accuracy of diagnostic methods relying on in-situ drive cycle and charge event analysis, combined with their value for improving onboard range estimation, could likely make them ubiquitous in PEVs of the near future. As the data could be used to great effect to support accurate resale value of a PEV, it may also become readily available to the vehicle owner. Thus, it would also likely be available to the repurposer to support valuation of packs prior to purchasing them.

\section{Use of Automotive Service Metrics to Identify Q1 Capacity}

Comparison of simulated automotive service statistics to calculated Q1 capacity fade has uncovered an excellent correlation of lifetime average battery temperature to Q1 for the BEV75s (Figure 14; R2 = 0.8686). For the PHEV20s, we find that this simple correlation between temperature and Q1 is significantly less compelling (R2 = 0.5169 ); however, the weighted addition of lifetime average SOC to lifetime average battery temperature results in a notable improvement (Figure 15, R2 = 0.6910).

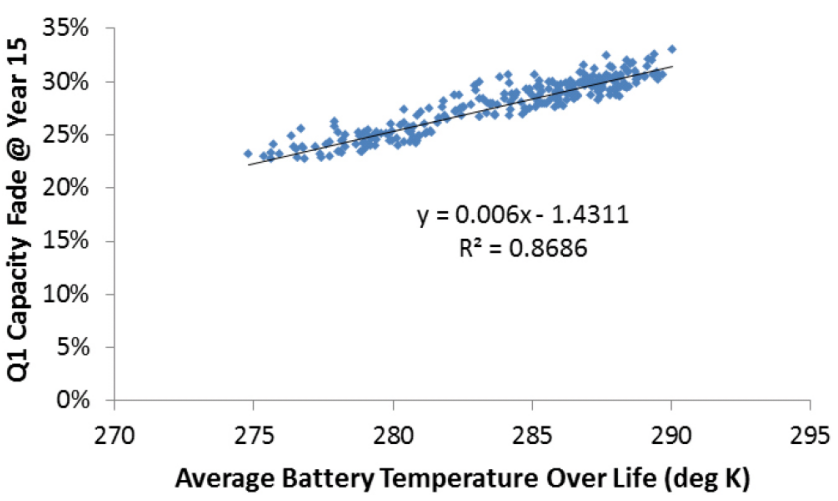

Figure 14. Correlation of Q1 capacity fade to average battery temperature for BEV75s

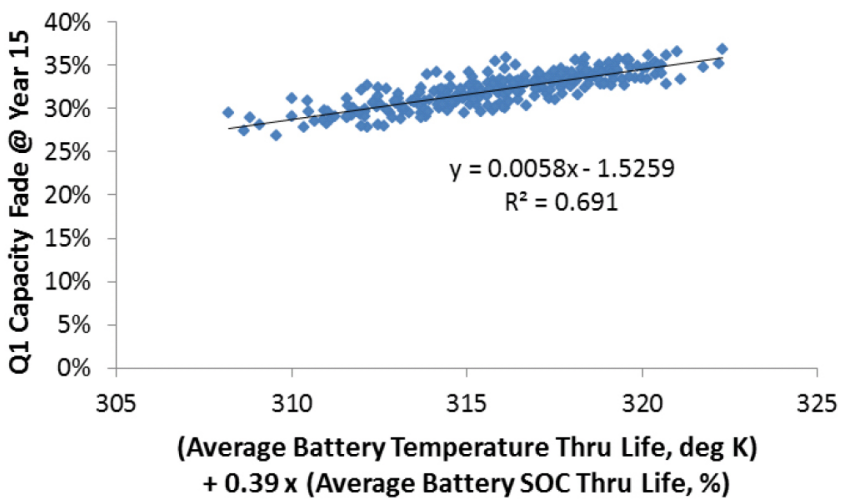

Figure 15. Correlation of Q1 capacity fade to average battery temperature and SOC for PHEV20s

Modern PEV batteries are equipped with the ability to record battery temperature and SOC. Compiling a lifetime average SOC would require minimal additions to on-board software if the vehicles do not already record and calculate such a metric. Compiling a lifetime average temperature metric, on the other hand, may require minor hardware additions. Temperature while parked is a large component of lifetime average temperature. While a battery's large thermal mass results in slow movement of battery temperatures and low required data logging rates, it is still necessary for the vehicle to "wake up" briefly and periodically during park events to measure and log new battery temperature data points.

The experience of the authors to date suggests that many PEVs do not record battery temperature data when the vehicle is in a key-off state and is not connected to a charger. Thus, software and possibly hardware changes would be required to implement these features. However, doing so would add a strong indicator of battery health and could be combined with the in-situ inverse modeling efforts described in the previous section for even more accurate estimates of battery SOH (possibly decoupling Q1, Q2, R1, and R2). As such, this feature too may be commonly included in PEVs in the coming years.

\section{Recommended Identification Strategies}

Taken together, the in-situ analysis of drive cycles and charging events along with recording of lifetime battery temperature and SOC metrics could provide an extremely high-confidence diagnostic for battery $\mathrm{SOH}$. It may even prove capable of decoupling different wear mechanisms, such as the R1, R2, Q1, and Q2 terms employed herein. Combined with additional on-board vehicle data of cell-level metrics to identify cell-to-cell variability (e.g., identifying faulty individual cells), and prognostic methods to predict future $\mathrm{SOH}$ trends (e.g., a battery life model), repurposers could make low risk purchasing decisions that maximize yield and minimize required in-house testing. Given the minimal cost anticipated to add these capabilities to the vehicles and to share the data with repurposers, this path is strongly recommended. However, given the long design cycle of new PEVs and the possibility that vehicle OEMs might not be willing to share such data, there may be a number of retired automotive batteries available without such information. Here, repurposing yields will certainly fall, and increased in-house testing will be required. 
Thus, the effects of both cases (with and without advanced vehicle data) were analyzed in an attempt to quantify the value of adding these capabilities to PEVs.

\section{CONCLUSIONS}

Herein we have presented a general methodology and tools for computing battery degradation during automotive and secondary use and translating battery wear to ascertain its effect on value. Under our assumed second use duty cycle of daily peak shaving, we have found that repurposed automotive batteries can last ten years or more in second use service when managed properly. In this role, capacity fade from automotive use has a much larger impact on second use value than resistance growth. Where capacity loss is driven by calendar effects more than cycling effects, average battery temperature during automotive service, which is often driven by climate, is found to be the single factor with the largest effect on the remaining value. Installing hardware and software capabilities onboard the vehicle that can both estimate remaining battery capacity from in-situ measurements, as well as track average battery temperature over time, will thereby help facilitate the second use of automotive batteries.

\section{REFERENCES}

1. Brooker, A., Thornton, M., and Rugh, J., "Technology Improvement Pathways to Cost-Effective Vehicle Electrification," presented at the SAE 2010 World Congress, April 13-15, 2010. Accessed December 12, 2014: http://www.nrel.gov/docs/fy10osti/47454.pdf

2. O'Keefe, M., Brooker, A., Johnson, C., Mendelsohn, M., Neubauer, J., and Pesaran, A., "Battery Ownership Model: A Tool for Evaluating the Economics of Electrified Vehicles and Related Infrastructure," presented at the 25th International Battery, Hybrid, and Fuel Cell Electric Vehicle Symposium \&Exposition, November 5-9, 2010. Accessed December 12, 2014: http://www.nrel.gov/docs/fy11osti/49127.pdf

3. "California Sets Energy Storage Target of $1.3 \mathrm{GW}$ by 2020," Green Tech Media. Accessed June 18, 2014: http://www.greentechmedia.com/ articles/read/california-sets-1.3gw-energy-storage-target-by-2020

4. "California Proposes First-In-Nation Grid-Scale Energy Storage Targets," Berkeley Energy \& Resources Collaborative. Accessed June 18, 2014: http://berc.berkeley.edu/california-proposes-first-in-nationgrid-scale-energy-storage-targets

5. "About the Self-Generation Incentive Program," California Public Utilities Commission. Accessed June 18, 2014: http://www.cpuc.ca.gov/ PUC/energy/DistGen/sgip/aboutsgip.htm

6. Wang, J., Liu, P., Hicks-Garner, J., Sherman, E., Soukiazian, S., Verbrugge, M., Tataria, H., Musser, J., and Finamore, P., "Cycle-Life Model for Graphite-LiFePO4 cells.” J. Power Sources 196(8):39423948, 2011. Accessed December 12, 2014: http://www.sciencedirect. com/science/article/pii/S0378775310021269

7. Peterson, S.B., Apt, J., and Whitacre, J.F., "Lithium-Ion Battery Cell Degradation Resulting from Realistic Vehicle and Vehicle-to-Grid Utilization." J. Power Sources 19(8):2385-2392, 2010.Accessed December 12, 2014: http://www.sciencedirect.com/science/article/pii/ $\underline{\mathrm{S} 0378775309017443}$

8. Schmalstieg, J., Kabitz, S., Ecker, M., and Sauer, D.U. "A Holistic Aging Model for Li(NiMnCo)O ${ }_{2}$ Based 18650 Lithium-Ion Batteries," J. Power Sources 257):325-334, 2014. Accessed December 12, 2014 : http://www.sciencedirect.com/science/article/pii/S0378775314001876

9. Christensen, J., and Newman, J., "A Mathematical Model for the Lithium-Ion Negative Electrode Solid Electrolyte Interphase," J. Echem. Soc. 151(11):A1977-A1988, 2004. Accessed December 12, 2014: http:// jes.ecsdl.org/content/151/11/A1977.full

10. Ploehn, H. J., Premanand, R., and White, R.E., "Solvent Diffusion Model for Aging of Lithium-Ion Battery Cells," J. Echem. Soc. 151(3):A456-A462, 2004. Accessed December 12, 2014: http://www. che.sc.edu/faculty/white $/ 2004$ solvent $\% 20$ diffusion $\% 20 \mathrm{hp} \% 20$ published. pdf
11. Safari, M., and Delacourt, C., "Simulation-Based Analysis of Aging Phenomena in a Commercial Graphite/LiFePO4 Cell," J. Echem. Soc. 158(12):A1436-A1447, 2011. Accessed December 12, 2014: http://jes. ecsdl.org/content/158/12/A1436.full.pdf

12. Smith, K., Earleywine, M., Wood, E., Neubauer, J. et al., "Comparison of Plug-In Hybrid Electric Vehicle Battery Life Across Geographies and Drive Cycles," SAE Technical Paper 2012-01-0666, 2012, doi:10.4271/2012-01-0666.

13. “Table 3.12: Car Scrappage and Survival Rates," Oak Ridge National Laboratory, 2006. Accessed November 19, 2014: http://cta.ornl.gov/data/ tedb33/Spreadsheets/Table3 12.xls

14. "2014 Chevrolet Limited Warranty and Owner Assistance Information." Chevrolet Motor Division, General Motors LLC, 2013. Accessed November 18, 2014: http://www.chevrolet.com/content/dam/Chevrolet/ northamerica/usa/nscwebsite/en/Home/Ownership/Warranty/02pdfs/2k14chev_lim_wm2ndprint.pdf

15. "Lithium-ion Battery Limited Warranty," Nissan USA. Accessed November 18, 2014: http://www.nissanusa.com/electric-cars/leaf/ charging-range/battery/.

16. "Nissan Announces LEAF Lease-Only Battery Replacement Program," PluginCars.com, June 21, 2013. Accessed November 18, 2014. http:// www.plugincars.com/nissan-announces-leaf-lease-only-batteryreplacement-program-127571.html

17. Neubauer, J., Brooker, A., and Wood, E., "Sensitivity of Plug-In Hybrid Electric Vehicle Economics to Drive Patterns, Electric Range, Energy Management, and Charge Strategies," J. Power Sources 236:357-364, 2013. Accessed December 12, 2014: http://www.sciencedirect.com/ science/article/pii/S0378775312011809

18. Neubauer, J., Brooker, A., and Wood, E., "Sensitivity of Battery Electric Vehicle Economics to Drive Patterns, Vehicle Range, and Charge Strategies," J. Power Sources 209:269-277, 2012. Accessed December 12, 2014: http://www.sciencedirect.com/science/article/pii/ $\underline{\mathrm{S} 0378775312005290}$

19. Neubauer, J., and Wood, E., "Thru-Life Impacts of Driver Aggression, Climate, Cabin Thermal Management, and Battery Thermal Management on Battery Electric Vehicle Utility," J. Power Sources 259:262-275, 2014. Accessed December 12, 2014: http://www. sciencedirect.com/science/article/pii/S0378775314002766

20. Neubauer, J., Battery Lifetime Analysis and Simulation Tool (BLAST) Documentation, NREL/TP-5400-63246, December 2014: http://www. nrel.gov/docs/fy15osti/63246.pdf

21. Neubauer, J., and Wood, E., "The Impact of Range Anxiety and Home, Workplace, and Public Charging Infrastructure on Simulated Battery Electric Vehicle Lifetime Utility,” J. Power Sources 257:12-20, 2014 Accessed December 12, 2014: http://www.sciencedirect.com/science/ article/pii/S0378775314000998

22. Neubauer, J., Wood, E., Burton, E., Smith, K., and Pesaran, A., FY14 Milestone: Simulated Impacts of Life-Like Fast Charging on BEV Batteries, NREL/MP-5400-63386, December 2014.Accessed January 13, 2015: http://www.nrel.gov/docs/fy15osti/63386.pdf

23. Traffic Choices Study - Summary Report. Seattle, WA: Puget Sound Regional Council, April 2008.

24. "Revisions and Additions to Motor Vehicle Fuel Economy Label." Government Printing Office. Federal Register Vol. 76, No. 129, July 6, 2011. Accessed November 12, 2013: http://www.gpo.gov/fdsys/pkg/FR2011-07-06/html/2011-14291.htm

25. Neubauer, J., Pesaran, A., Williams, B., Ferry, M. et al., “A TechnoEconomic Analysis of PEV Battery Second Use: Repurposed-Battery Selling Price and Commercial and Industrial End-User Value," SAE Technical Paper 2012-01-0349, 2012, doi:10.4271/2012-01-0349.

26. “Average Mean Temperature Index by Month." Earth System Research Laboratory/National Oceanic \& Atmospheric Administration. Accessed November 11, 2012: http://www.esrl.noaa.gov/psd/data/usclimate/tmp. $\underline{\text { state. } 19712000 . \text { climo }}$

\section{CONTACT INFORMATION}

Jeremy Neubauer

D.Sc. Senior Engineer

National Renewable Energy Laboratory

jeremy.neubauer@nrel.gov 


\section{ACKNOWLEDGMENTS}

This study was supported by Dave Howell and Brian Cunningham of the Energy Storage, Vehicle Technologies Office, Office of Energy Efficiency and Renewable Energy, U.S. Department of Energy. Special thanks to Mike Ferry and the California Center for Sustainable Energy second-use team for continued consultation and analysis of EV deployments.

\section{DEFINITIONS/ABBREVIATIONS}

${ }^{\circ} \mathbf{C}$ - degrees Celsius

B2U - Battery Second Use

BEV - battery electric vehicle

BEV75 - battery electric vehicle with a 75-mile range

BLAST - Battery Lifetime Analysis and Simulation Tool

BLAST-V - Battery Lifetime Analysis and Simulation Tool for Vehicles

BOL - beginning of life

DOD - depth of discharge

NREL - National Renewable Energy Laboratory

OEM - original equipment manufacturer

PEV - plug-in electric vehicle

PHEV - plug-in hybrid electric vehicle

PHEV20 - plug-in hybrid electric vehicle with a 20 mile range

PVT - present value of throughput

Q1 - capacity loss due to calendar effects

Q2 - capacity loss due to cycling effect

R1 - resistance growth due to calendar effects

$\mathbf{R} 2$ - resistance growth due to cycling effects

SOC - state of charge

SOH - state of health

All rights reserved. No part of this publication may be reproduced, stored in a retrieval system, or transmitted, in any form or by any means, electronic, mechanical, photocopying, recording, or otherwise, without the prior written permission of SAE International. 